P111 (continued)

Objective: To improve the Expanded Food and Nutrition Education Program (EFNEP) nutrition intervention by learning about Latino children's perceptions of physical activity (PA), screen time and sugar-sweetened beverages (SSBs) using focus group data.

Theory, Prior Research, Rationale: This study used the health belief model as its theoretical foundation. Research has strongly linked increased consumption of SSBs and sedentary lifestyles to obesity/overweight in youth. Improved understanding of the determinants will inform interventions to promote healthy lifestyles.

Description: Target audience was Latino children in grades K-8. Latino children who completed a 6-week series of EFNEP intervention $(n=19)$ participated in focus groups and responded to open-ended questions. Focus group questions addressed perceived benefits and barriers to PA, screen time and beverage consumption. All sessions were audio recorded and transcribed verbatim.

Evaluation: Transcribed data were subject to open-coding thematic analyses. Several key themes emerged from this study. Many children view PA as a means to get healthy and be more active. Children were aware of which drinks were unhealthy and felt that parents should either not purchase them or find a way to keep them out of sight. Many children said that screen time offers entertainment and that screen time activities make them 'feel good'. Some children noted that devices make them lazy, unfocused and unable to concentrate.

Conclusions and Implications: Focus groups with EFNEP youth participants provide in-depth insights into children's perceptions of PA, screen time and beverage consumption, thus, allowing us to tailor nutrition education programs to the needs of program participants.

Funding: USDA

\section{P112 Growing Healthy Kids: Feasibility of a Garden-Based Nutrition Education Intervention for Low-Resource Families}

JeffreyLaubert, laubert.3@osu.edu, The Ohio State University, 453 West 10th Avenue, Columbus, $\mathrm{OH} 43210$; Ashlea Braun, $R D N, L D$, The Ohio State University; Pat Bebo, MS; Mary-Jon Ludy, PhD, RDN, FAND, Bowling Green State University; Ingrid Adams, PhD, RDN, $L D, L D E$, The Ohio State University; Colleen Spees, PhD, MEd, RDN, $L D, F A N D$

Objective: To determine the feasibility and acceptability of a theory-driven and evidence-based summer nutrition education and feeding program for low-resource families in the Midwest.

Study Design, Setting, Participants, Intervention: A 10-week feasibility pilot was completed in the summer of 2016 targeting children, ages 8-11 years, and their parent/adult caregivers (PAC) from low-resource communities (as defined by school SNAP-eligibility). Program components included: group nutrition education and cooking demonstrations led by Cooperative Extension
Agents; weekly produce harvesting at a rural and an urban community garden; and remote motivational interviewing coaching (e-MIC) facilitated by trained registered dietitians.

Outcome Measures and Analysis: Feasibility and acceptability were measured by weekly child/PAC attendance logs, pre- to post- behavior surveys, post-program evaluations, and PAC engagement logs recorded by the e-MIC.

Results: A total of 30 children/PAC's were recruited during a 3-week period. Group class and harvesting retention were $>85 \%$. Further, over $65 \%$ of PACs actively engaged with the e-MIC. Post-programmatic surveys for the children documented improvements in consumption of fruits and vegetables. PAC surveys showed improved utilization of MyPlate for meal planning, greater confidence in eating healthy on a budget, increased family exposure to new produce, and more adventurous eating among the family.

Conclusions and Implications: The results of this pilot support the feasibility and acceptability of a comprehensive garden-based nutrition education and feeding program for low-resource families. Future studies will focus on efficacy.

Funding: None

\section{P113 iCook 4-H: Parenting Typology Reflected in Youth Created Cooking Videos}

Sa'Nealdra Wiggins, BS, University of Tennessee; Sarah Colby, PhD, RD, scolby1@utk.edu, University of Tennessee, 1215 West Cumberland Avenue, 229 Jessie Harris Building, Knoxville, TN 37916; Chelsea Allison, BS, University of Tennessee; Rochelle Butler, PhD;

Magen Payne; Ainsley Ellington; Hillary N. Fouts, PhD;

Lauren Moret, PhD; Melissa D. Olfert, MS, RDN, LD, DrPH, West Virginia University; Kendra K. Kattelmann, $P h D, R D$, LN, FAND, South Dakota State University; Lisa FranzenCastle, $P h D, M S, R D$, University of Nebraska-Lincoln; Adrienne A. White, $P h D, R D$, University of Maine

Objective: Using a mixed-methods approach to assess differences in parenting typologies observed in youth-created cooking videos from the iCook $4-\mathrm{H}$ project.

Study Design, Setting, Participants, Intervention: The iCook program was a randomized, control-treatment, 5-state intervention designed for 9-10 year olds and their adult meal preparers. Through a series of eight sessions, participants learned to cook, eat, and play together. Participants used provided video cameras to create cooking videos reflecting what they learned in six sessions.

Outcome Measures and Analysis: After training was conducted and $>80 \%$ inter-rater reliability achieved, videos $(\mathrm{n}=172)$ were narrated and coded using NVivo by teams of two independent researchers with a third resolving any differences. Code frequencies were analyzed for relationships by typology using ANOVA. 
P113 (continued)

Results: Four typologies of parents were observed: Supportive, Negative, Laid-back, and Mixed. Parents with Negative and Supportive typologies had more affluent kitchen equipment whereas parents with Laid-back and Mixed typologies had less affluent kitchen equipment $(\mathrm{p}=0.014)$. Parents with Negative typologies had the least cluttered kitchen environments; those with Mixed typologies had the most cluttered environments $(\mathrm{p}<$ 0.000). Youth whose parents were Laid-back or Mixed typology asked for assistance more often than those with Supportive or Negative typology parents $(\mathrm{p}<0.000)$. Youth of parents with Supportive typology had the highest levels of cooking confidence whereas youth of parents with Laid-back typology had the lowest levels of cooking confidence $(\mathrm{p}=0.001)$.

Conclusion and Implications: Cooking-related parenting typologies were uniquely identified in this research. Impact of differing parenting typology on youth health-related behavior warrants further investigation.

Funding: USDA

\section{P114 Impact of a Mindfulness-Based Program on Child Diet and Eating Behaviors}

Lynn Brann, PhD, RD, FAND, lbrann@syr.edu, Syracuse University, 559 White Hall, Syracuse, NY 13244;

Rachel Razza, PhD, Syracuse University; Dessa BergenCico, PhD

Objective: To examine the impact of a mindfulnessbased program (MBP) on child dietary intake and eating behaviors.

Study Design, Setting, Participants, Intervention: A six-week mindful eating and yoga intervention to promote children's self-regulation was conducted in two childcare centers (intervention $n=24$; control $n=20$ ) with children 3-5 years old.

Outcome Measures and Analysis: Pre- and post-intervention, parents completed three-day child food records and the Child Eating Behavior Questionnaire (CEBQ) to assess factors such as food responsiveness and satiety responsiveness. Children's Eating in the Absence of Hunger (EAH) was measured pre- and post-intervention. Outcome evaluation analyses included paired t-tests, McNemar tests, independent samples t-tests and Mann Whitney U tests.

Results: Children's diets were low in key nutrients of concern including fiber, vitamin $\mathrm{D}$, vitamin $\mathrm{E}$ and potassium and were high in sodium. The intervention group had significantly higher intakes of several nutrients compared to the intervention group at the pre-assessment; however, both groups consumed below recommendations for vitamin $\mathrm{D}$ and potassium and above for sodium. No significant difference were found between groups for dietary intake at the post-assessment. There were no significant differences in the subscales of the CEBQ between the intervention and control groups at the pre- and post-assessment. After hunger was neutralized, there were no differences in the number of calories consumed within or between groups of children for EAH.

Conclusions and Implications: This pilot study did not show a significant impact of utilizing a MBP on child dietary intake and eating behaviors. Further research needs to evaluate success of MBPs with a larger sample size and greater involvement of parents is necessary.

Funding: Falk College Research Center at Syracuse University

\section{P115 Increasing Children's Fruit and Vegetable Consumption Using Nutrition Education and Active Choice Principles}

Robyn M. Cafiero, MS, robyncafiero@gmail.com, Montclair State University, 253 Park Avenue, Rutherford, NJ 07070; Yeon Bai, PhD, RD, Montclair State University; Doreen Liou, EdD, RD; Charles Feldman, PhD

Objective: To increase children's fruit and vegetable consumption using nutrition education and active choice principles.

Study Design, Setting, Participants, Intervention: The study used pre-/post-intervention comparison design. The intervention utilized a dual module of the Social Cognitive Theory (SCT) and active choice (AC) for second graders in one school in Northern, NJ. For nine months, the intervention group received a combination of nutrition education and AC; the control group received AC. AC allowed the participants to choose between two fruits and/or vegetables.

Outcome Measures and Analysis: Variables measured included fruit/vegetable preference, consumption, reciprocal determinism, self-efficacy, behavioral capability, and modeling from SCT. Paired/independent t-tests, chisquare analyses were employed to compare groups.

Results: Eighty-nine students participated in the study (intervention $=46$, control $=43$ ): $57 \%$ female and $43 \%$ males; mean age 7.64 (SD 0.48). No change was observed in fruit consumption. Vegetable consumption and preference differed after intervention between groups: for consumption (self-efficacy), 6.5\% increase for intervention and $4.5 \%$ decrease for control $(\mathrm{p}=.01)$; for preference, $4.3 \%$ increase for intervention and $2.4 \%$ decrease for control $(\mathrm{p}=.57)$. Similarly, knowledge (behavior capability) of fruit/vegetables increased $4.3 \%$ in intervention, but decreased $2.4 \%$ in control $(\mathrm{p}=.14)$. All students were engaged within AC even if they did not take a fruit/vegetable that day.

Conclusions and Implications: Nutrition education combined with AC had a positive impact on knowledge and vegetable consumption/preference in the intervention group. A longer invention duration with multiple locations may grow the study validity for innovative program to be implemented throughout school districts nationwide.

Funding: Jennifer Layne Acupuncture and Wellness; ShopRite of Rochelle Park, NJ 